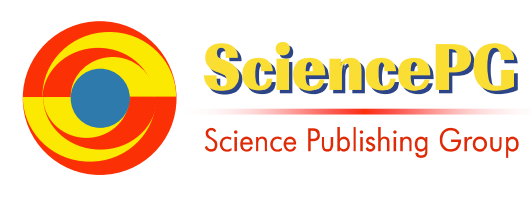

\title{
An Algorithm for a Sub-Nyquist Rate AM and FM Software-Defined Radio Based on the Market Paradigm
}

\author{
Thomas KokumoYesufu, Joel Adeniyi Otolorin, Akinbode Alex Olawole
}

Department of Electronic and Electrical Engineering,ObafemiAwolowo University, Ile-Ife, Nigeria

\section{Email address:}

thomas_yesufu@yahoo.com (T.K. Yesufu), otolorinjoel@yahoo.com(J.A. Otolorin), alex_olawole@yahoo.com (A.A. Olawole)

\section{To cite this article:}

Thomas KokumoYesufu, Joel AdeniyiOtolorin, Akinbode Alex Olawole. An Algorithm for a Sub-Nyquist Rate AM and FM SoftwareDefined Radio Based on the Market Paradigm. Science Journal of Circuits, Systems and Signal Processing.Vol. 4, No. 3, 2015 , pp. 18-22. doi: $10.11648 /$ j.cssp.20150403.11

\begin{abstract}
Software-defined radio accomplishes both modulation and demodulation processes using software. While this has a number of advantages, which includes flexibility, interoperability, sustainability, and adaptability, the requirement for sampling the signal for digital processes toward adequate recovery often involves the use of a fast but expensive analogue-todigital converter (ADC). This, in a way translates to higher cost and requirement for bigger storage. This paper presents a method of switched signal recovery at uniform sampling rates that are less than the frequently over-estimated Nyquist rate employed. In particular, an algorithm for achieving this was implemented for an AM wave, under-sampled at varied uniform rates up-to twice the carrier rate, and then demodulated using the Market Paradigm. Furthermore,the slope detectorwas also implemented by including a differentiator after the sampling stage of the algorithm. The simulated results showed that the algorithm was able to recover the message signal at sampling rates far less than twice the carrier rate without the need for any additional hardware. Specifically, the best value of the Spurious Free Dynamic Range (SFDR) obtained for the recovered message signal was $20 \mathrm{~dB}$ at a sampling rate of less than $20 \%$ of the Nyquist rate for the carrier signal.
\end{abstract}

Keywords: Software-Defined Radio, Sampling, Big Data, Market Paradigm, Agent-Based Detection, Wireless Networks

\section{Introduction}

A radio is any kind of device that wirelessly transmits or receives signals in the radio frequency (RF) part of the electromagnetic spectrum in order to facilitate the transfer of information. In today's world, radios exist in a multitude of items such as cell phones, computers, vehicles and television systems (Sharma, 2009).

In a traditional radio, all the signal processes such as frequency translation, filtering, demodulation, etc., are implemented usinghardware. Althoughthis approach has proven to be practical for a very wide range of applications, there are cases in which the ability to alter radio functionality at run-time is highly desirable, but cannot be accomplished without altering the hardware design and structure. Interoperability with the existing legacy systems, ability to operate with region- specific communication standards, and readiness for future communication protocols are few of the benefits for desiring a reconfigurable system. Traditional radio lacks the capability to accomplish these plans without changing the hardware and many times the structure too, and thus results in higher production cost and minimal flexibility in supporting multiple waveform standards (Lehr et al., 2002). Traditional radio systems, subsequently, lacks the flexibility to adapt to newer standards or to change the relative proportion of transceiver channel modules without changing all the hardware.

Software-defined radio (SDR) was proposed by Mitola III (1992) to signal the shift from hardware designed dominated radio systems to systems where major part of the functionality is defined in software. SDR is characterized as a radio whose physical layer behaviour can be significantly altered through changes to its software (ShajedulHassan and Balister, 2005). It uses software to modulate and demodulate radio signals (Millhaem, 2006). While this has a number of advantages, which include flexibility, interoperability, sustainability, and adaptability, the requirement for sampling the signal at twice its maximum frequency to fulfill Shannon sampling theorem (Nyquist criterion) has to be met for adequate recovery. Consequently, this often involves the use of a fast but expensive analogue-to-digital converter (ADC), which in a way translates to higher cost and requirement for bigger storage. The major motivation of this theorem lies in the 
ability to shift processing tasks from the analog to the digitalor switched domain. Digital signal processing (DSP) is therefore a major driving force that supports the wide popularity of sampling at the Nyquist rate (Proakis and Manoulis, 1992; and Mishali and Eldar, 2010 and 2011). Following this, conversion speeds that are twice the signal's maximum frequency have recently become more and more difficult to obtain, andconsequently, alternatives to high rate sampling are expected to continue to draw considerable attention in both academia and industry (e.g., Kumar and Reddy, 2014). It is also important to note that sampling, as a solution, must be explored in line with the laws of large numbers (e.g., Ross, 2009) in order to successfully transmit and receive a message over a channel.

Sampling below the Nyquist rate otherwise known as subNyquist sampleing have prominent advantages over the conventional Nyquist theorem in that the DSP operations are carried out at low input rate, and storage may not require a preceding compression stage. Demodulation is one of the methods used to achieve this. In the method, the incoming signal is multiplied by the carrier frequency of a band of interest, so as to shift contents to the origin, and then sampled uniformly in time. This technique however needs analog preprocessing (Proakis and Salehi, 1995) that adds to the hardware complexity. Under-sampling refers to uniform sampling of bandpass signals at a rate lower than the maximum frequency. In this technique, uniform sampling of a bandpass input $\mathrm{x}(\mathrm{t})$ whose information band lies in the frequency range $\left(f_{u}, f_{l}\right)$ are sampled at a rate of $f_{S}$ that obeys:

$$
\frac{2 f_{u}}{k} \leq f_{S} \leq \frac{2 f_{l}}{k-1}
$$

for some integer $1 \leq k \leq f_{u} / B$, (where $k=1$ corresponds to Nyquist rate). This ensures that aliases of the positive and negative contents do not overlap for sampling frequency greater or equal to twice the bandwidth of the signal, i.e, $f_{S}$ $=2 \mathrm{~B}$ (Landau, 1967; and Vaughan et al., 1991).However,apart from the fact that a significanthigher rate is likely to be required in practice to cope with design imperfections due to possibledeviations in the values of $f_{s}$, $f_{l}, f_{u}$ not every ADC device fits an under-sampling system: only those devices whose front-end analog bandwidth exceedsfu are viable.

Periodic non-uniform sampling on the other hand allows for minimal rates that approach values equal to the bandwidth of the signal without complicated analog preprocessing (Kohlenberg, 1953; and Lin and Vaidyanathan, 1998). But the method adds to the hardware complexity as a set of time-delay elements are required beside the ADC.

This paper presents a method of signal recovery at sampling rates that are less than the Nyquist rate for switched signals commonly associated with sampled data systems, and AM and FM signals. In particular, an FM wave would be sampled at uniform rate, and then the intelligence will be recovered and other advantages indicated.

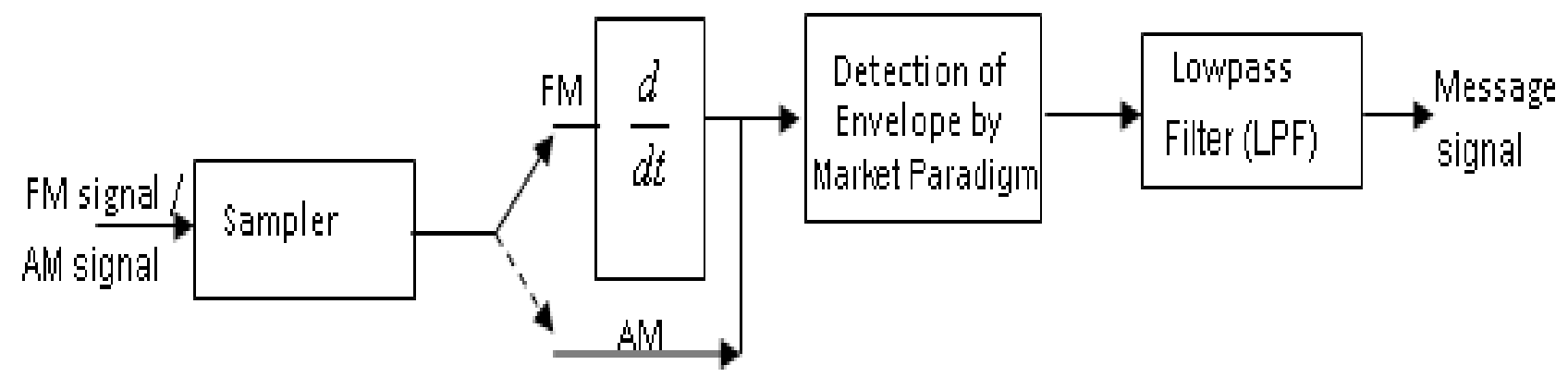

Figure 1. Model for Proposed Software-Based Demodulation of FM and AM Signals.

\section{Theoretical Development}

Considera generalized modulated or switched signalshown in Figure 1 and expressed as

$$
S(t)=A_{C}(t) \cos \left(2 \pi f_{C} t+\phi(t)\right)
$$

$A_{C}(t)$ is the instantaneous amplitude, which is assumed constant for an FM signal. For AM signal: $A_{C}(t)=A_{C}+m(t)$. $\phi(t)$ is the phase, which also for an AM is assumed constant, but for FM signal: $\phi(t)=K_{f} \int_{0}^{t} m(t) d t$ while $m(t)$ is the message signal.

The generalized modulated signal represented by (2) is sampled uniformly at the rate of

$$
T_{S}=\frac{n}{r\left(f_{C}+h f_{m}\right)}
$$

where $n, r$ and $h$ are integers ( $r=2$ corresponds to the Nyquist rate, $h=1$ for narrow band FM with only one (1) pair of sidebands), and the maximum frequency deviation is $2 h f_{m}$ (forwideband FM signal with $\mathrm{h}$ significant pairs of sidebands).

For $f_{C} \gg f_{m}$, we can equally assume that:

$$
T_{S}=\frac{n}{r f_{C}}
$$

without loss of generality, and therefore, 


$$
S[n]=A \cos \theta\left(n T_{S}\right) \sum_{n=-\infty}^{\infty} \delta\left(t-n T_{S}\right)
$$

for an FM wave;

$$
S[n]=A_{C}\left(n T_{S}\right) \cos \theta \sum_{n=-\infty}^{\infty} \delta\left(t-n T_{S}\right)
$$

for an AM wave.

Use is normally made of a slope detector, a frequency demodulation technique, which can readily combine FM and AM demodulation processes with a suitable modification in hardware.A simple approximation of the slope detector is an ideal differentiator followed by an envelope detector. The slope detector produces an output that may be assumed to be proportional to the instantaneous frequency deviation of the input signal.Therefore using the slope detector method of demodulation affords us the possibility of using a common sampler and differentiator circuit for both FM and AM signals (Figure 1).

The Market Paradigm is an algorithm developed to detect the envelope of sampled AM signal. In the analysis of interacting agents' scenario, such as market-mediated economic interactions or strategic interactions in game theoretic settings with few agents, as explained by Yesufu and Yesufu (2003) and Yesufu and Oladimeji (2008), it has been realized that the market paradigm is a good tool for analyzing time series data, strategic system analysis and network analysis. Hence, the series, $X_{t}$, is a general form for a stationary series given by

$$
X_{t}=r_{t} \cdot X_{t-1}+e_{t}
$$

where $e_{t}$, the error term, has zero mean and covariance, and a constant variance and $r_{t}$, the root of the series, has a value of unity for random walk. Accordingly, we have

$$
X_{t-1}=X_{t}-\frac{1}{2}\left(X_{t} \cdot X_{t-1}\right)\left(X_{t}\right)
$$

On rearranging (7), we have

$$
X_{t}=\frac{2\left(X_{t}-X_{t-1}\right)}{X_{t} \cdot X_{t-1}}
$$

By comparing (6) with (8) for convergence, we have

$$
r_{t}=2 \cdot \frac{\left(X_{t}-X_{t-1}\right)}{\left(X_{t}\right)\left(X_{t-1}\right)^{2}}
$$

The value of $r_{\mathrm{t}}$ indicates the motivation or amount of information present by adding a new term, $X_{t}$, to the series $\mathrm{S}$ after the term $X_{t-1}$. In other words this is the characteristic root of the time series at the time t. $\mathrm{S}[\mathrm{n}]$ is a discrete time signal, and so the differentiation operation for the FM wave was done in digital domain, using backward difference equation,

$$
S^{\prime}[\mathrm{n}] \approx \frac{1}{T_{s}}\left[S\left(n T_{s}\right)-S(n-1) T_{s}\right]
$$

The instantaneous frequency $f_{i}(t)$,

$$
f_{i}(t)=f_{c}+\Delta f m(t)=\theta^{\prime}(t)
$$

is proportional to the difference between consecutive data samples. The envelope of the differentiated output $S^{\prime}[\mathrm{n}]$ was detected using market paradigm as in (10). The output of the differentiator is a time series. The message can be extracted by a low pass filtering (Otolorin, 2013). The summary of the algorithm is as shown in Table 1 .

Table 1. Algorithm for the demodulation of AM and FM signals based on the market paradigm.

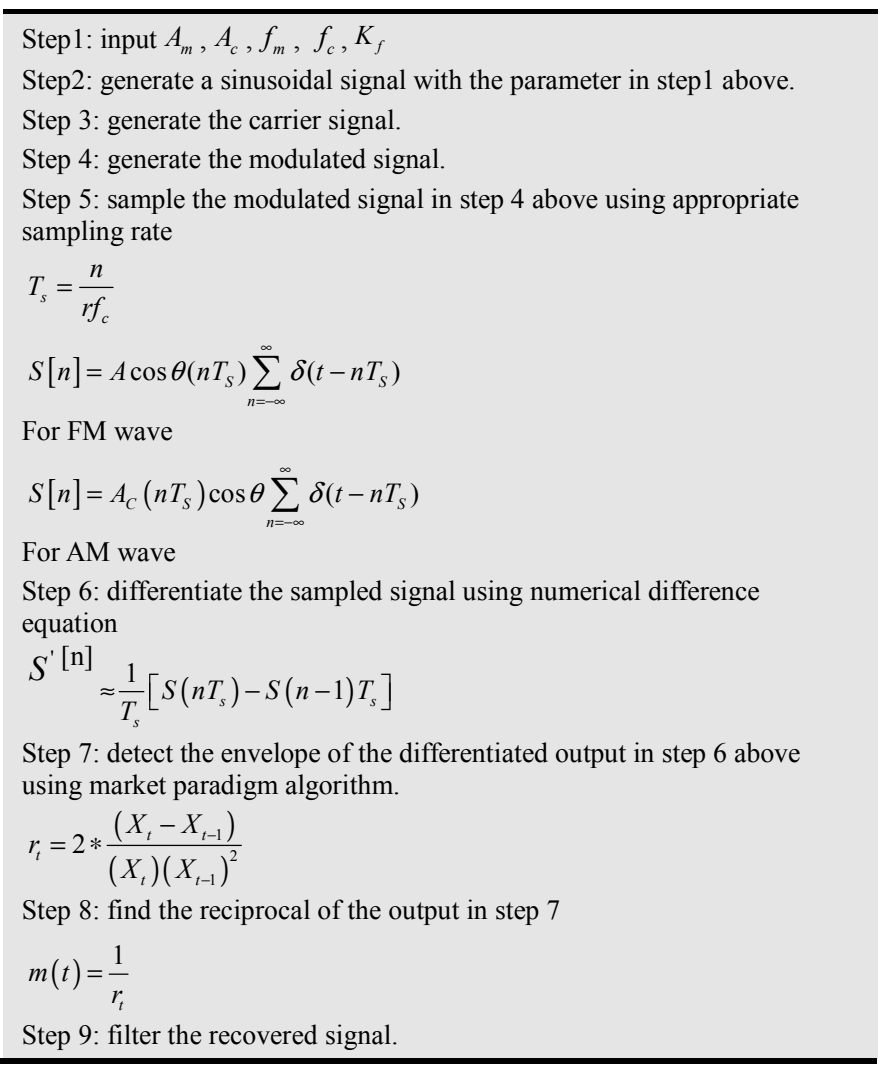

Table 2. SFDR Values in dB for Final Output of Demodulated AM signal at Different Sampling Rates.

\begin{tabular}{llll}
\hline $\begin{array}{l}\text { Sampling } \\
\text { Rate, } \mathbf{f}_{\mathrm{s}}(\mathbf{H z})\end{array}$ & $\begin{array}{l}\text { Normalized Value } \\
\text { of Baseband Signal } \\
\text { Amplitude, BB }\end{array}$ & $\begin{array}{l}\text { Normalized } \\
\text { Value of } \mathbf{S H}_{\text {peak }}\end{array}$ & SFDR in dB \\
\hline $0.1 \mathrm{f}_{\mathrm{c}}$ & 0.18883962 & 0.131096738 & 3.170024641 \\
$0.2 \mathrm{f}_{\mathrm{c}}$ & 0.19273360 & 0.145999306 & 2.412132955 \\
$0.3 \mathrm{f}_{\mathrm{c}}$ & 0.00552357 & 0.005293273 & 0.369907719 \\
$0.4 \mathrm{f}_{\mathrm{c}}$ & 0.19844341 & 0.025300372 & 17.89019548 \\
$0.5 \mathrm{f}_{\mathrm{c}}$ & 0.00485271 & 0.001198534 & 12.14668133 \\
$0.6 \mathrm{f}_{\mathrm{c}}$ & 0.01107818 & 0.010611077 & 0.374174837 \\
$0.8 \mathrm{f}_{\mathrm{c}}$ & 0.00101180 & 0.027836617 & -28.790482 \\
\hline
\end{tabular}




\begin{tabular}{|c|c|c|c|}
\hline $\begin{array}{l}\text { Sampling } \\
\text { Rate, } \mathbf{f}_{\mathrm{s}}(\mathbf{H z})\end{array}$ & $\begin{array}{l}\text { Normalized Value } \\
\text { of Baseband Signal } \\
\text { Amplitude, } \text { BB }_{\text {peak }}\end{array}$ & $\begin{array}{l}\text { Normalized } \\
\text { Value of } \mathrm{SH}_{\text {peak }}\end{array}$ & SFDR in $\mathrm{dB}$ \\
\hline $1.0 \mathrm{f}_{\mathrm{c}}$ & 0.00485872 & 0.001211051 & 12.06719343 \\
\hline $1.5 \mathrm{f}_{\mathrm{c}}$ & 0.01631337 & 0.020656931 & -2.05044048 \\
\hline $2.0 \mathrm{f}_{\mathrm{c}}$ & 1.00000000 & 0.125454018 & 18.03030846 \\
\hline
\end{tabular}

Table 3. SFDR Values in dB for Final Output of Demodulated FM signal at Different Sampling Rates.

\begin{tabular}{llll}
\hline $\begin{array}{l}\text { Sampling } \\
\text { Rate, } \mathbf{f}_{\mathrm{s}}(\mathbf{H z})\end{array}$ & $\begin{array}{l}\text { Normalized Value of } \\
\text { Baseband Signal } \\
\text { Amplitude, BB }\end{array}$ & $\begin{array}{l}\text { Normalized } \\
\text { Value of } \\
\text { MSH }_{\text {peak }}\end{array}$ & SFDR in dB \\
\hline $0.1 \mathrm{f}_{\mathrm{c}}$ & 0.000000173 & 0.000000057 & 9.6869 \\
$0.2 \mathrm{f}_{\mathrm{c}}$ & 0.000000438 & 0.000000776 & -4.9590 \\
$0.3 \mathrm{f}_{\mathrm{c}}$ & 0.000034172 & 0.000051506 & -3.5638 \\
$0.4 \mathrm{f}_{\mathrm{c}}$ & 0.000821177 & 0.000078208 & 20.4237 \\
$0.5 \mathrm{f}_{\mathrm{c}}$ & 0.000184884 & 0.000042799 & 12.7094 \\
$0.6 \mathrm{f}_{\mathrm{c}}$ & 0.000136323 & 0.000158879 & -1.3300 \\
$0.8 \mathrm{f}_{\mathrm{c}}$ & 0.002799009 & 0.042900221 & -23.7091 \\
$0.9 \mathrm{f}_{\mathrm{c}}$ & 0.081096614 & 0.042170239 & 5.6799 \\
$1.0 \mathrm{f}_{\mathrm{c}}$ & 0.003854422 & 0.002940377 & 2.3511 \\
$1.5 \mathrm{f}_{\mathrm{c}}$ & 0.646000000 & 0.572968293 & 1.0392 \\
$2.0 \mathrm{f}_{\mathrm{c}}$ & 1.000000000 & 0.084895802 & 21.4223 \\
\hline
\end{tabular}

\section{Results and Discussion}

This work considered both AM and FM demodulation based on sub-Nyquist sampling rate. We have assumed a single tone modulated system. The message frequency was chosen to be $20 \mathrm{~Hz}$, while the carrier waveform has frequency of $2 \mathrm{kHz}$. Other parameters were chosen to generate the baseband, carrier and FM signal waves. Modulation indices of 0.1 to 1.2 were tested by varying the frequency deviation constant, $K_{f}$ between 0 and 5 while keeping the amplitude of modulating signal constant. The generated $\mathrm{FM}$ wave equation was sampled at $0.1 \mathrm{f}_{\mathrm{c}}$ to $1 \mathrm{f}_{\mathrm{c}}, 1.5 \mathrm{f}_{\mathrm{c}}$ and $2 \mathrm{f}_{\mathrm{c}}$.

The performance of the developed algorithm was evaluated using the Spurious Free Dynamic Range (SFDR) of the recovered message signal. At each sampling rate, the normalized amplitude at frequency of the baseband signal $\left(\mathrm{BB}_{\text {peak }}\right)$ and that of the most significant harmonic $\left(\mathrm{MSH}_{\text {peak }}\right)$ were determined and tabulated as shown in Tables 2 and 3 for AM demodulation and FM demodulation processes respectively. The corresponding values of SFDR (dB) were computed and the graph of the sampling rate against the SFDR (dB) was plotted for the two cases as shown in Figures2 and 3, respectively. It can be observed from Figure 4 that $0.4 \mathrm{f}_{\mathrm{c}}$ is the optimum sampling rate for both FM and AM. Hence, a sampler at $0.4 \mathrm{f}_{\mathrm{c}}$ (that is at $20 \%$ of Nyquist) can be used for both FM and AM waves.

The market paradigm suitably uses the slope detection method of FM demodulation to achieve interoperability between AM and FM, as well as other sampled data systems. The results show that obtaining the conveyed intelligence in carrier switched systems, which have been processed to meet certain transmission properties, will definitely require that sampling should be done at rates that are far less than the carrier rate but at least twice the highest frequency in the message signal. The ploy in modulation is to switch a signal at a given carrier rate, which is to achieve the channel- efficient transfer of information by a reversible means to take a sample of the population (i.e., considerably reduce the sample size required to represent a message signal) before transmitting it.

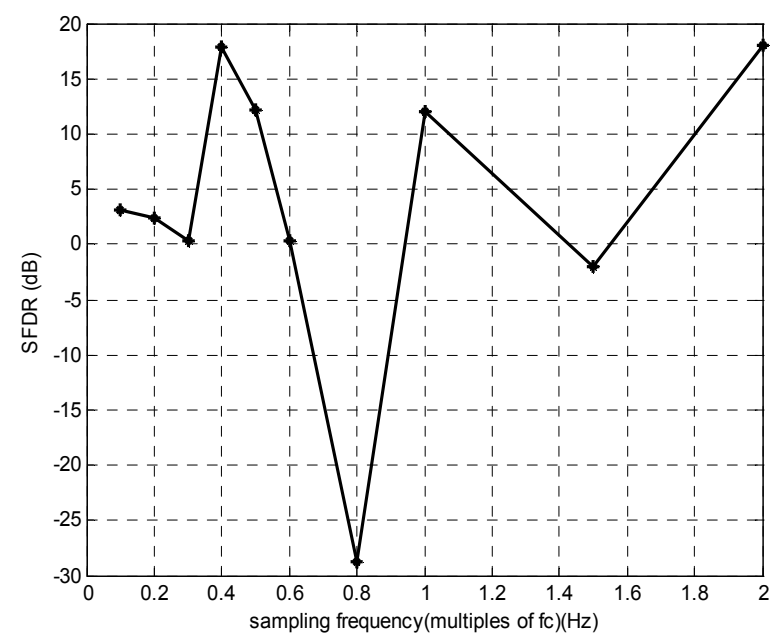

Figure 2. Plot of SFDR for Demodulated AM Signal.

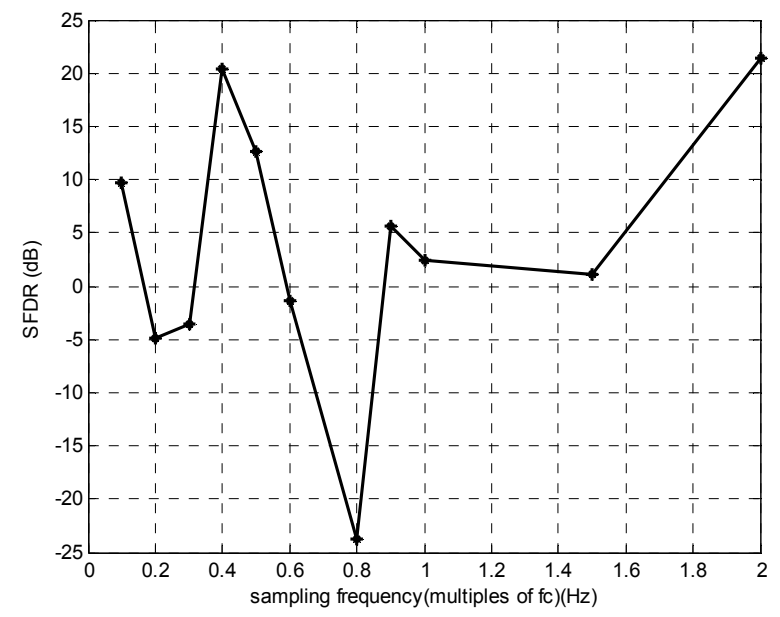

Figure 3. Plot of SFDR (db) for Final Output of Demodulated FM Signal.

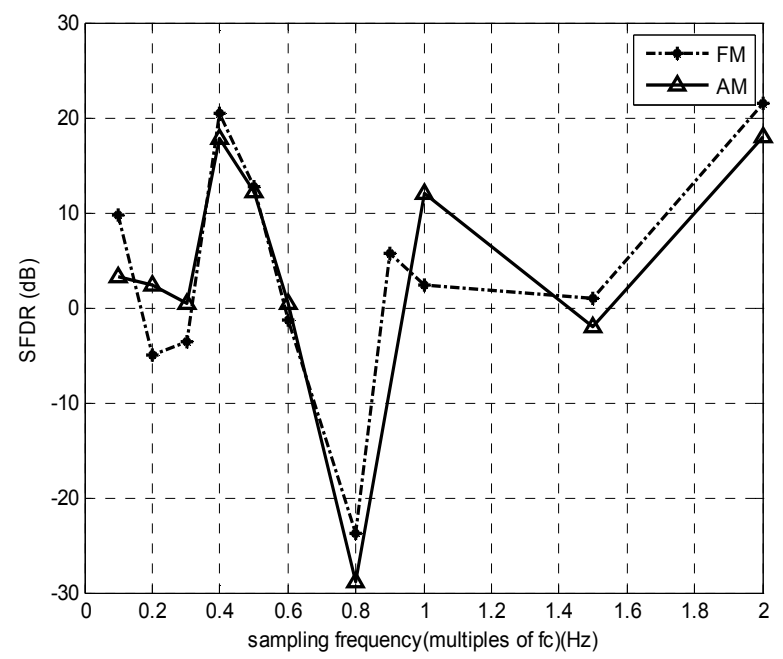

Figure 4. Plot of SFDR (dB) for Demodulated FM and AM Signals. 


\section{Conclusion}

This paper presented the development of an algorithm for the sub-Nyquist rate sampled data processing of AM and FM signals. In this case, the sampling rate was found to be appropriate, being focused on the message signal rather than the carrier signal. This was possible because AM and FM signals are switched and processedwaveforms that have inherent features of small-sized but representative random samples of a population of nodes in a wireless network. The entire population, in this case, is the intelligence to be recovered from the AM and FM signals. This is consistent with the law of large numbers as well as with the ability of the Market Paradigm to effectively detect the envelope of an AM waveform at sampling rates well below the carrier rate. The developed algorithm is suitable for the emergence of cheaper software-defined radio receivers and more secured wireless networks based on the possibility of using a much lower sample size for faster intrusion detection and other related implementation of cleverer schemes in digital radios.

\section{References}

[1] Kohlenberg, A. (1953): "Exact interpolation of band-limited functions," J. Appl. Phys., vol. 24, pp. 1432-1435.

[2] Kumar, G.V.P and Reddy, D.K. (2014): An Agent Based Intrusion Detection System for Wireless Network with Artificial Immune System (AIS) and Negative Clone Selection, 2014 International Conference on Electronic Systems, Signal Processing and Computing Technologies (ICESC), pp. $429-433$.

[3] Landau, H. J. (1967): "Necessary density conditions for sampling and interpolation of certain entire functions," Acta Math., vol.117, pp. 37-52, Feb. 1967.

[4] Lehr,W., Merino, F., and Gillet, S. E. (2002): "Software Radio: Implementation for Wireless Services, Industry Structure and Public Policy”, Massach.

[5] Lin, Y. P. and Vaidyanathan, P. P., "Periodically nonuniform sampling of bandpass signals," IEEE Trans. Circuits Syst. II, vol. 45 , no. 3, pp. 340-351, Mar. 1998

[6] Millhaem, M. (2006): "Software shapes next-generation RF instrumentation (radio frequency, software-defined radio)". Wireless Design and Development Publication, Available Online:http://www.highbeam.com/doc/ 1G1- 155474366.html

[7] Mishali, M. and Eldar, Y.C. (2010): "From Theory to Practice: Sub-Nyquist Sampling of Sparse Wideband Analog Signals", IEEE Journal of Selected Topics in Signal processing, Vol. 4, No. 2, pp. 375-390.
[8] Mishali, M. and Eldar, Y.C. (2011): Sub-Nyquist Sampling Bridging theory and practice, IEEE Signal Processing Magazine, Vol. 28 No. 6, pp. 98 - 124.

[9] Mitola III, J. (1992): Software Radios- Survey, Critical Evaluation and Future Directions, In Telesystem Conference NTC-92, pp. 15-23.

[10] Otolorin, J.A. (2013): Development of an Algorithm for Implementing a Low Cost Frequency Modulated (FM) Receiver, Unpublished M.Sc. Thesis, Department of Electronic and Electrical Engineering, ObafemiAwolowo University, Ile-Ife, 101p.

[11] Olademeji, O. O. (2008): "Development of a New Algorithm for the Implementation of High Precision Delta-Sigma Digital-to-Analog Converter", Unpublished M.Sc. Thesis, Department of Electronic and Electrical Engineering, ObafemiAwolowo University, Ile-Ife., pp. 64 - 73

[12] Proakis, J. G. and Manolakis, D. G. (1992): Digital Signal Processing: Principles, Algorithms, and Application. Macmillan, New York, pp. 395 - 467.

[13] Proakis, J.G. and Salehi, M. (1994): "Communication Systems Engineering", Prentice-Hall International, Inc. New Jersey, pp 297, 328- 385.

[14] Ross, S. (2009). A first course in probability (8th ed.). Prentice Hall Press.

[15] Sharma, S. P. (2009): Basic Radio and Television,Tata McGraw-Hill, New Delhi, pp. 330-371

[16] ShajedulHasan, S.M., and Balister, P. (2005): "Prototyping a Software Defined Radio ReceiverBased on USRP and OSSIE ”, Chameleonic Radio Technical Memo No. 1, pp. 1, 8.

[17] Vaughan, R. G., Scott, N. L., and White, D. R. (1991): "The theory of bandpass sampling," IEEE Trans. Signal Processing, vol. 39, No.9, pp. 1973-1984.

[18] Yesufu, O.A. and Yesufu, T.K. (2003): Development of the Market Paradigm for Analyzing Systems, Social Science Research Network (SSRN) Electronic Journals of Agriculture and Natural Resource Economics, Dispute \& Conflict Resolution, Econometrics, Strategy \& Economics, Development Economics, Risk Management, Environmental Economics, Available Online through http://www.ssrn.com/abstract=437181.

[19] Yesufu, T.K. and Oladimeji, O. O. (2008): An Algorithm for High Precision Delta-Sigma Digital-to-Analog Converters, In: Dagli, C.H., D.L. Encke, K.M. Bryden, H. Ceylan, and M. Gen (Eds.): Intelligent Engineering Systems Through Artificial Neural Networks, Vol. 18, ASME Press Series, New York, pp. 595 - 600 . 